\title{
Enucleación esofágica de leiomioma asistida por robot: reporte de caso y revisión de la literatura
}

\section{Robotic-assisted enucleation of an esophageal leiomyoma: A case report and review of literature}

\author{
Miguel Ricardo Buitrago RamíRez, MD ${ }^{1}$ Jorge Ramírez Pereira, MD²
}

Diego Fernando Salcedo Miranda, MD ${ }^{3}$

\section{Resumen}

Los leiomiomas esofágicos son tumores benignos resecados por enucleación esofágica cuando son mayores de $5 \mathrm{~cm}$ o en lesiones de cualquier tamaño que sean sintomáticas o con sospecha de malignidad. Tradicionalmente, la resección se ha realizado por técnica de enucleación abierta; sin embargo, la cirugía mínimamente invasiva surgió como una técnica con grandes ventajas, especialmente la tecnología robótica. Se reporta un caso de leiomioma esofágico del esófago medio con enucleación mediante el uso de una técnica de toracoscópica asistida por robot. La esofagoscopia intraoperatoria y transiluminación fueron complementos útiles para identificar el esófago y desarrollar un plano de disección extramucosa seguro que, junto con la asistencia robótica, parecen minimizar los riesgos intraoperatorios; entre ellos, la probabilidad potencial de lesión de la mucosa, así como también mejorar el tiempo de recuperación posoperatoria.

Palabras clave: leiomioma esofágico, tumor benigno, robótica, enucleación, cirugía mínimamente invasiva, toracoscopia.

\begin{abstract}
Esophageal leiomyomas are benign tumors, which are resected by esophageal enucleation when they are larger than $5 \mathrm{~cm}$, or in lesions of any size that are symptomatic or suspicious of malignancy. Resection has traditionally been performed by the technique of open enucleation; however, minimally invasive surgery has emerged as a technique with great advantages, and especially robotic technology seems to offer advantages. We report a case of esophageal leiomyoma of the middle esophagus with enucleation using a robot-assisted thoracoscopic technique. Intraoperative esophagoscopy and transillumination were useful adjuncts to identify the esophagus and to develop an extra mucosal dissection plane which with robotic assistance appear to minimize intraoperative risks among them, potentially the possibility of mucosal injury, and improving postoperative recovery time.
\end{abstract}

Key words: esophageal leiomyoma, benign tumor, robotic, enucleation, minimally invasive surgery, thoracoscopy.

\footnotetext{
${ }^{1}$ Cirujano de Tórax, Universidad El Bosque, Clínica de Marly. Bogotá, Colombia.

${ }^{2}$ Fellow de Cirugía de Tórax, Universidad El Bosque. Bogotá, Colombia.

Residente de Cirugía General, Universidad El Bosque. Bogotá, Colombia.

Autor de correspondencia

Diego Fernando Salcedo Miranda. Correo electrónico: dsalcedo_ub@hotmail.com. Recibido: 4/10/17. Aceptado: 30/11/17.
} 


\section{Introducción}

Los tumores del tracto gastrointestinal (GIST) son las lesiones benignas más frecuentes de este tracto. Los tumores benignos del esófago son poco frecuentes, y el leiomioma es el más común de estos tumores, que representa más del $80 \%(1,2)$. Se origina de la capa circular muscular del esófago distal, esófago medio y, particularmente, en la unión gastroesofágica. Sus dimensiones van desde $1 \mathrm{~cm}$ hasta $29 \mathrm{~cm}$, según se reporta en la literatura, y es el tipo de lesión ideal para cirugía de preservación de órgano.

Convencionalmente, los leiomiomas esofágicos se han resecado mediante toracotomía abierta; sin embargo, han surgido técnicas toracoscópicas sujetas a las habilidades del cirujano, e inclusive métodos asistidos por robot, que dan el beneficio de la imagen tridimensional (3D), disminuyendo el riesgo de perforación de la mucosa esofágica y mejorando la disección de las estructuras, por lo que probablemente se convertirá en una ventaja frente a otros procedimientos quirúrgicos en un futuro. Nosotros presentamos un caso de enucleación de leiomioma esofágico por robot con transiluminación intraoperatoria con esofagoscopia, facilitando la disección segura del plano extramucoso y logrando la preservación de órgano $(2,3)$.

\section{Material y métodos}

Presentación de un caso de enucleación de leiomioma esofágico por toracoscopia izquierda asistida por robot en una clínica de Bogotá, Colombia, con revisión de la literatura actual sobre el tema.

\section{Presentación del caso}

Se trata de una mujer de 41 años, con antecedentes de disfagia y evidencia, por escanografía de tórax (TC), de una lesión esofágica de 4,1 cm (Figura 1); a quien en el año 2013 se le intentó realizar resección por toracoscopia; sin embargo, el procedimiento se cambió a toracotomía debido a un tumor de mediastino que comprometía la pared del esófago torácico. Se tomó biopsia compatible con fascículos de músculo liso sin pleomorfismo nuclear ni mitosis o necrosis compatible histopatológicamente con leiomioma benigno del esófago. Por crecimiento de la masa, en el año 2017 se realizaron estudios de extensión; entre ellos, un esofagograma preoperatorio con visualización de una lesión de $4,1 \mathrm{~cm}$ que produjo alteración del tránsito esofágico (Figura 2); también se confirmó masa submucosa por ultrasonografía endoscópica (Figura 3) de aproximadamente 3,6 cm en su eje mayor, a lo largo de la pared anterior del esófago en el tórax superior, inferior a la vena ácigos, apareciendo como masa hipovascular sin adenopatías paraesofágicas, como lo muestra la ecoendoscopia. La resección del tumor se consideró necesaria debido al crecimiento gradual de la lesión.

Bajo anestesia general, y utilizando un tubo de doble lumen en la posición de decúbito lateral izquierdo,

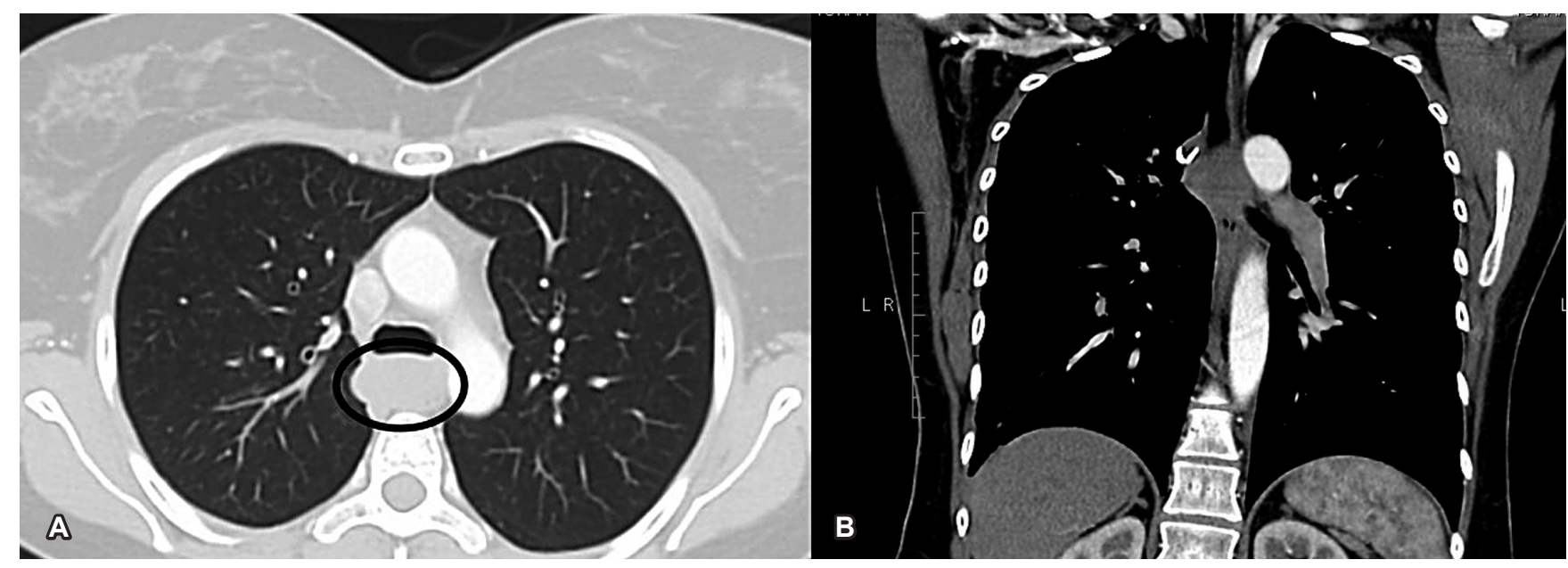

Figura 1. A y B. TC con contraste. Evidencia de una masa esofágica heterogénea con medidas de $4,1 \mathrm{~cm}$ en dos proyecciones. 


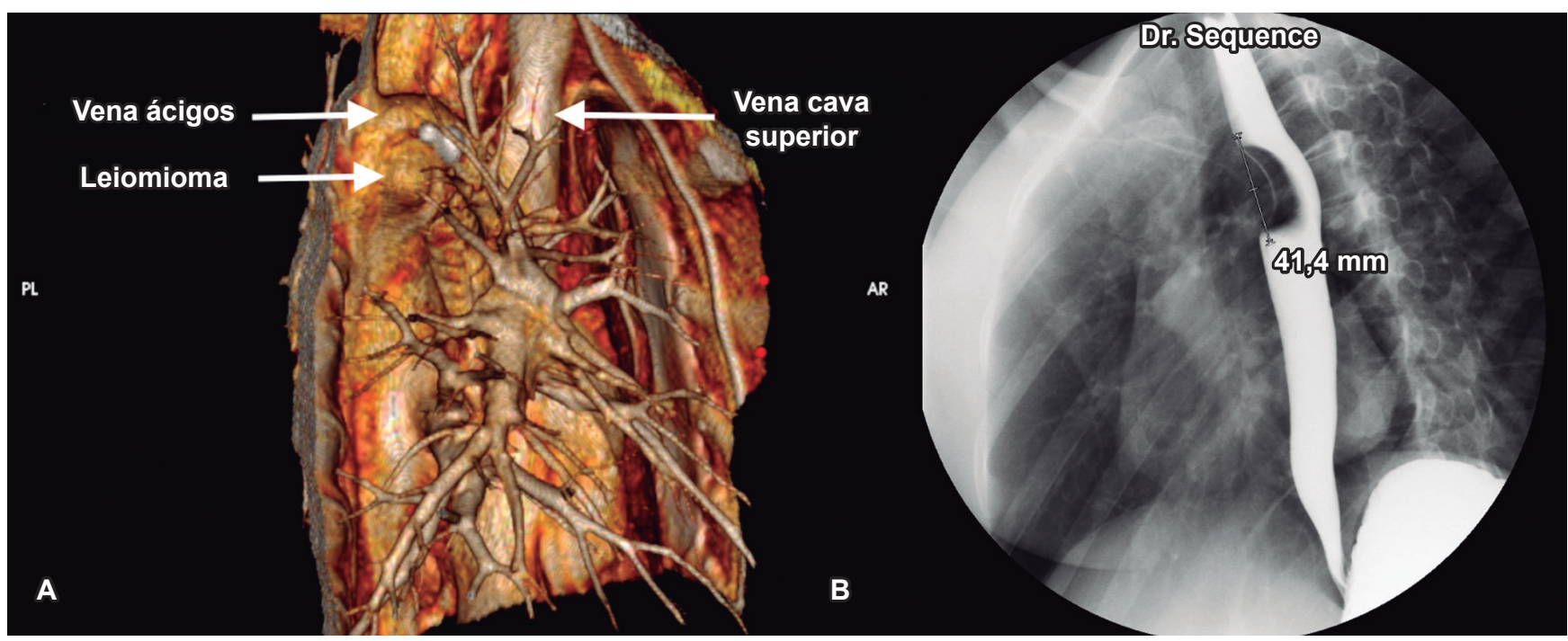

Figura 2. A. Reconstrucción en 3D, donde se muestra la ubicación anatómica de la lesión. B. El esofagograma muestra un defecto de llenado en el esófago con superficie mucosa lisa intacta.

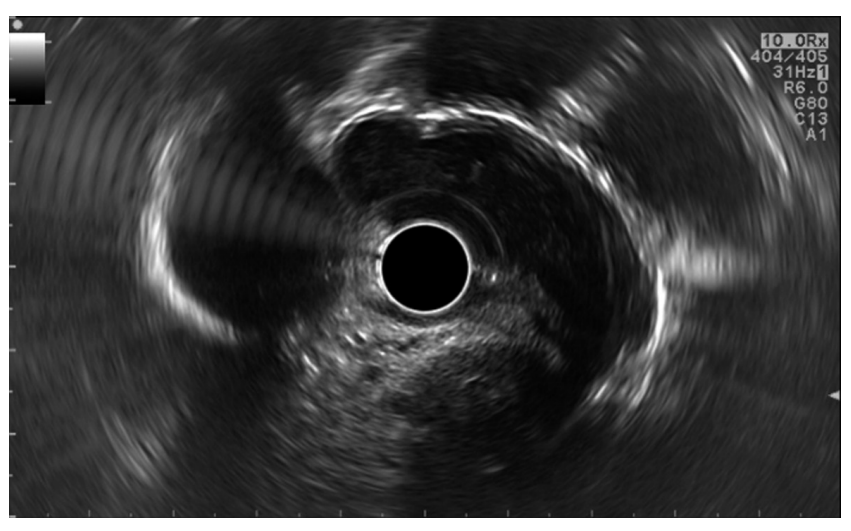

Figura 3. Ultrasonografía endoscópica del esófago (EUS) que revela la masa hipoecoica bien circunscrita originada en la capa muscular propia.

se aisló el pulmón derecho y se colocaron trocares: un trocar de robot de $8 \mathrm{~mm}$ (Intuitive Surgical) se colocó en la línea axilar media por encima de la quinta costi1la. Se insertó una cámara en este trocar, mientras que otros puertos se ubicaron bajo visualización directa. A continuación, se colocó un trocar de robot de $8 \mathrm{~mm}$ (Intuitive Surgical) a $1 \mathrm{~cm}$ lateral a la columna vertebral sobre la octava costilla. Un tercer trocar de robot de $8 \mathrm{~mm}$ (Intuitive Surgical) se implantó a $8 \mathrm{~cm}$ del último trocar sobre la octava costilla. A continuación, se ubicó un puerto de $12 \mathrm{~mm}$ Endopath Xcel (Ethicon,
New Jersey) a $8 \mathrm{~cm}$ del último trocar sobre la octava costilla, cerca de la línea axilar posterior. Finalmente se colocó un trocar de $12 \mathrm{~mm}$ Endopath Xcel (Ethicon) a $8 \mathrm{~cm}$ inferior del puerto de la cámara sobre el diafragma. Luego el robot se acopló con brazos de robot conectados a Thoracic Grasper (Intuitive Surgical) en el puerto de la izquierda a la cámara, Cadiere Robot Forceps (Intuitive Surgical) en el puerto derecho a la cámara y Permanent Cautering Spatula (Intuitive Surgical) en el puerto izquierdo a la cámara.

El tumor se identificó en el lado de la cabeza del arco ácigos a través de la pleura mediastinal (Figura 4A). La pleura mediastínica, adventicia del esófago y capa muscular propia se seccionaron longitudinalmente para exponer la cápsula tumoral (Figura 4B). El tumor parecía surgir principalmente de la lámina muscular de la mucosa. Un tumor redondo, elástico y duro se enucleó con éxito por disección casi roma sin lesión de la mucosa (Figura 4C). Después de la extirpación del tumor, la pleura mediastínica, adventicia del esófago y muscular propia del esófago se cerraron con suturas continuas (Figura 4D) y se colocó un tubo de tórax.

El tubo de tórax se retiró el día 1 posoperatorio y se realizó esofagograma que reveló mucosa esofágica lisa intacta sin fugas o estenosis (Figura 5). La paciente 

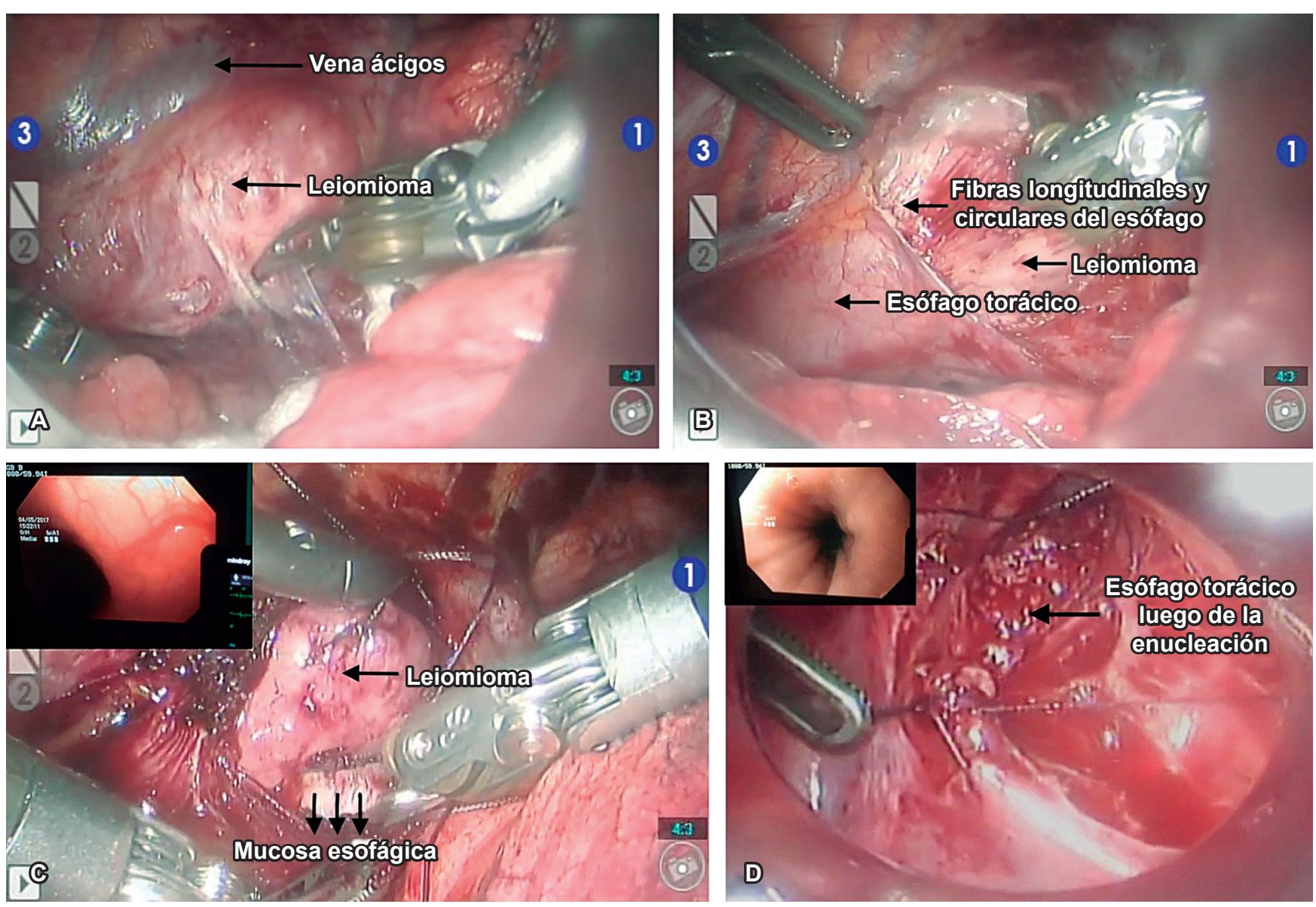

Figura 4. A. Imagen del leiomioma cubierto por pleura mediastínica del esófago antes de la disección. B y C. Visualización del leiomioma luego de la disección, con la inspección esofagoscópica durante la cirugía, asegurando la completa escisión del tumor. D. Integridad de la mucosa después de la enucleación.

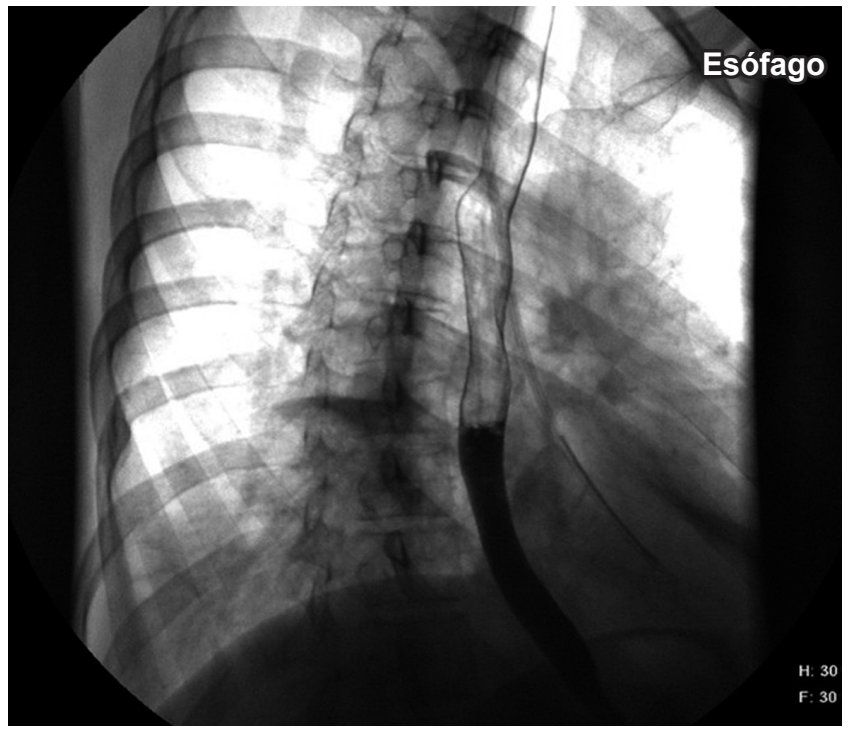

Figura 5. Esofagograma posoperatorio: evidencia de integridad del esófago torácico sin muestra de estenosis y fístulas. inició ingesta por vía oral a partir de ese mismo día. El curso después de la cirugía fue favorable y se dio egreso en esas primeras 24 horas. El examen histopatológico del espécimen resecado mostró células tumorales en forma de huso (Figura 6), que parecían difusamente positivas para desmina, pero negativas para DOG-1, c-KIT, CD34 y S100 en el examen de inmunohistoquímica. El diagnóstico patológico final fue leiomioma del esófago sin evidencia de malignidad.

\section{Discusión}

Los tumores del tracto gastrointestinal (GIST) son las lesiones benignas más frecuentes de este tracto. El leiomioma es el tumor mesenquimal benigno más común, y representa más del $80 \%$ de los tumores esofágicos benignos (1). Este puede ocurrir a cualquier edad, pero presenta un pico de incidencia entre la tercera $y$ 


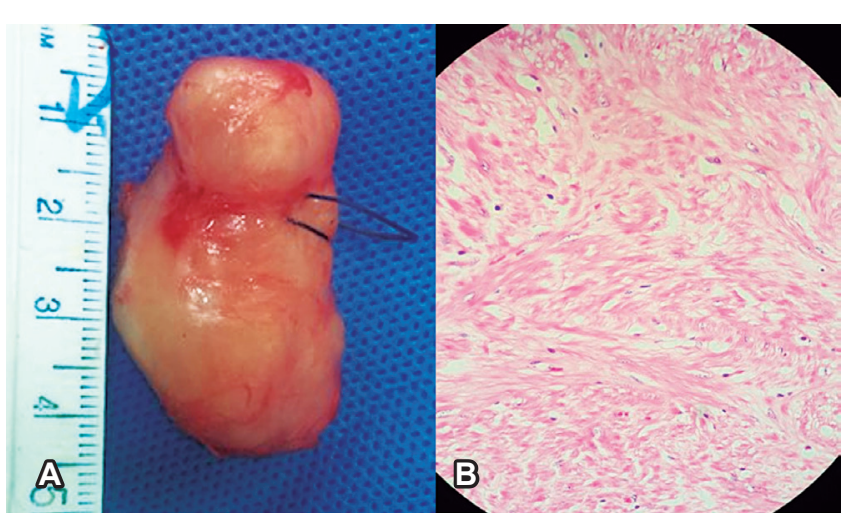

Figura 6. A. Pieza quirúrgica del leiomioma después de la enucleación. Masa de $4 \mathrm{~cm}$ con seudocápsula intacta. B. Vista microscópica del tumor. H/E x40 (inserción H/E x200).

quinta década de vida. Usualmente, su localización se presenta en el tercio medio e inferior del esófago (2). Estos tumores son intramurales, tratándose de un tumor de crecimiento lento; el tamaño suele ser menor de $5 \mathrm{~cm}$ (4). La mitad de los pacientes son asintomáticos, y en aquellos sintomáticos, la disfagia y malestar epigástrico son los síntomas más frecuentes; sin embargo, también pueden ocurrir regurgitación, sangrado gastrointestinal alto y pérdida de peso $(4,5)$.

Una vez se realiza el hallazgo, la esofagogastroscopia combinada con ultrasonografía endoscópica del tumor es obligatoria para excluir cáncer de esófago como diagnóstico diferencial. El aspecto típico de los GIST y leiomioma es de lesión homogénea e hipoecoica con margen claro, originada en la musculatura propia o muscular de la mucosa. Estos se pueden diferenciar de otras lesiones benignas del esófago, como los lipomas que son hiperecoicos y pueden distinguirse fácilmente, aunque algunos reportes identifican calcificaciones en $1,8 \%$ de los tumores $(6,7)$. Debido a que esta característica parece ser exclusiva de los leiomiomas esofágicos, una TC con contraste puede descartar afección extraluminal de la masa y excluir la posibilidad de malignidad $(8,9)$.

Se debe realizar biopsia de la masa esofágica antes de la cirugía para distinguir entre GIST o leiomioma esofágico, aunque las guías de la NCCN (National Comprehensive Cancer Network) no sugieren realizar biopsia preoperatoria de las masas resecables, debido a que los GIST pueden ser blandos y frágiles, y la biopsia puede causar hemorragia e incrementar el riesgo de diseminación del tumor por ruptura de la seudocápsula, e incluso, la localización submucosa y la biopsia por aguja fina (FNA) pueden ser insuficientes para diferenciar entre el leiomioma y la variante maligna $\operatorname{GIST}(1,4)$.

Las opciones de manejo se basan en el tamaño de las lesiones: para lesiones pequeñas $(<2 \mathrm{~cm})$ y asintomáticas se debe establecer un consenso sobre la indicación de cirugía y el mejor método de tratamiento. Algunos investigadores recomiendan la evaluación por ecografía endoscópica cada 1 o 2 años. Las razones de este enfoque son las siguientes: el leiomioma es un tumor de crecimiento lento, la transformación maligna es extremadamente rara, y el trauma quirúrgico puede ser más perjudicial para el paciente $(4,10,11)$. Sin embargo, las lesiones grandes ( $>5 \mathrm{~cm}$ ) o sintomáticas, sin importar el tamaño, se debe resecar, considerando la enucleación quirúrgica, como en el caso de nuestra paciente con una lesión de 4,1 cm, pero sintomática (12).

Las opciones de manejo del leiomioma esofágico incluyen: enucleación por toracotomía o endoscópica, inyección de alcohol, enucleación por videotoracoscopia y asistida por robot, e incluso el surgimiento de la disección endoscópica submucosa tunelizada (ESTD) para la resección de tumores submucosos del esófago $(4,13)$. Sin embargo, debido a que no existen criterios formales para la selección del enfoque quirúrgico, la elección del procedimiento de resección del tumor depende de las habilidades del operador $(4,8)$. Incluso, en casos de leiomiomatosis esofágica difusa o leiomioma esofágico gigante, a menudo se requiere de una esofagectomía (8).

Durante mucho tiempo, la enucleación esofágica del leiomioma vía abierta fue el estándar de manejo de los tumores benignos, pero con la aparición del enfoque mínimamente invasivo se ha visto un descenso en el porcentaje de efectos adversos, como dolor y tiempo de estancia hospitalaria, comparado con los procedimientos abiertos $(2,3,10)$. Adicionalmente, estas técnicas mínimamente invasivas son más exigentes que la cirugía abierta debido a la visión bidimensional, la coordinación reducida entre los ojos y manos, y la disminución de los grados de libertad al realizar los movimientos (5). 
El valor agregado que brinda los sistemas asistidos por robot en procedimientos mínimamente invasivos de resección toracoscópica del tumor esofágico se detecta en la disección a lo largo de estructuras vitales, como la vena pulmonar, vena ácigos, aorta o tráquea, logrando una visión tridimensional, mejorando la coordinación de los ojos y manos del cirujano, y disminuyendo el riesgo de perforación de la mucosa esofágica $(5,14)$.

La guía endoscópica intraoperatoria se ha convertido en una herramienta útil en la enucleación debido a que puede identificar la integridad de la mucosa, ya que la complicación posoperatoria más frecuente se da por la laceración de la mucosa (15). La evidencia de una mucosa integra permite un inicio más temprano de la vía oral, por lo cual algunos autores sugieren que la esofagoscopia intraoperatoria es esencial para el desempeño de la enucleación de tumores submucosos benignos del esófago.

Esta técnica proporciona un procedimiento seguro y preciso, y disminuye el riesgo de complicaciones posoperatorias (16). Por ello decidimos realizar un abordaje asistido por robot con guía endoscópica intraoperatoria. La desventaja de este procedimiento es que se requiere de la disponibilidad del equipo y el entrenamiento especializado del grupo médico involucrado.

El abordaje asistido por robot se asocia con una menor incidencia de lesión de la mucosa. La toracoscopia robótica asistida, en particular en enucleación de tumor benigno esofágico, proporciona una distinción más clara entre las capas anatómicas por la visión estereoscópica 3D ampliada superior, minimizando así las posibilidades de perforación de la mucosa, en comparación con la videotoracoscopia (VATS), y sobre el mediastino, evitando la mortalidad quirúrgica y la pérdida de sangre intraoperatoria (17-19). Se ha demostrado reducción de la estancia hospitalaria debido a la disminución del dolor posoperatorio, disminución de las fugas por laceración de la mucosa y reincorporación más rápida a las actividades diarias (15). Las ventajas se hacen más pronunciadas cuando la lesión se encuentra hacia el extremo del esófago torácico; es decir, el esófago torácico superior y hacia el hiato donde se encuentran estructuras vitales.
La cirugía esofágica asistida por robot está entrando en un campo prometedor; sin embargo, aún faltan estudios y evidencia que soporten un enfoque robótico en comparación con otros. Por supuesto, los tiempos quirúrgicos prolongados y los altos costos que requiere este abordaje quirúrgico son algunos de los obstáculos para una amplia aceptación de esta técnica. Se necesitan investigaciones adicionales en el campo de la enucleación de lesiones esofágicas por robot para recomendar esta práctica en comparación con otras técnicas mínimamente invasivas $(17,19)$. En nuestro caso se ofreció a la paciente un abordaje asistido por robot por la ubicación de la lesión y el antecedente quirúrgico, en la que no se presentaron complicaciones intraoperatorias ni posoperatorias.

La distinción preoperatoria de leiomioma y GIST es difícil por lo mencionado anteriormente; por tanto, la evaluación patológica de la pieza quirúrgica con hematoxilina eosina e inmunohistoquímica es el método de referencia para el diagnóstico de cualquier leiomioma. Estas lesiones son CD34 y CD117 negativa, sin mutaciones c-KIT y son positivas para la desmina y actina de músculo liso (SMA), mientras que los GIST suelen ser, aunque no siempre, negativos; estos presentan un patrón de inmunohistoquímica diferente, alrededor del 95\% es positivo para KIT (CD117), 60\%-70\% para CD34, 30\%-40\% para SMA y 5\% para la proteína S-100 $(1,6,7)$.

Para finalizar, el principal método de seguimiento de los pacientes luego de la enucleación de tumores benignos del esófago aún no se ha establecido. En algunos casos de GIST, el seguimiento regular se recomienda con base en la probabilidad de recurrencia de la lesión, pero a diferencia del GIST, la recurrencia de otros tipos de tumores esofágicos, como el leiomioma, es muy rara, por lo que puede no ser necesario realizar seguimiento oncológico para estos tumores, aunque otros recomiendan un seguimiento clínico periódico (20).

\section{Conclusiones}

La enucleación de leiomioma esofágico con asistencia robótica permite la resección completa de la lesión; además, es segura y tiene beneficios, como incremento de la precisión en la resección, disminución del 
riesgo de perforación esofágica y del trauma quirúrgico, logrando una reducción de la estancia hospitalaria en comparación con los abordajes abiertos, siendo más visible en la resección de lesiones complejas cercanas a estructuras importantes del tórax y mediastino. Adicionalmente, la cirugía asistida por robot ofrece ventajas como mejor exposición de las estructuras, visión 3D, magnificación de la imagen y mejor coordinación de los ojos y manos del cirujano; sin embargo, faltan estudios que soporten este abordaje sobre la toracoscopia. A pesar de esto se ha establecido que este enfoque es factible y eficaz en el tratamiento quirúrgico de leiomiomas del esófago.

\section{Aprobación ética}

Se obtuvo el consentimiento informado por escrito de la paciente para la publicación de este documento.

\section{Conflicto de intereses}

Los autores no tienen conflicto de intereses potencial con respecto a la publicación de este documento.

\section{Referencias}

1. Jiang P, Jiao Z, Han B, Zhang X, Sun X, Su J, et al. Clinical characteristics and surgical treatment of oesophageal gastrointestinal stromal tumours. Eur J Cardiothorac Surg. 2010;38(2):223-7. DOI: 10.1016/j.ejcts.2010.01.040.

2. Kernstine KH, Andersen ES, Falabella A, Ramirez NA, Anderson CA, Beblawi I. Robotic fourth-arm enucleation of an esophageal leiomyoma and review of literature. Innovations (Phila). 2009;4(6):354-7. DOI: 10.21037/amj.2017.01.15.

3. DeUgarte DA, Teitelbaum D, Hirschl RB, Geiger JD. Robotic extirpation of complex massive esophageal leiomyoma. J Laparoendosc Adv Surg Tech A. 2008;18(2):286-9. DOI: 10.1089/lap.2007.0067.

4. Choi SH, Kim YT, Han KN, Ra YJ, Kang CH, Sung SW, et al. Surgical management of the esophageal leiomyoma: lessons from a retrospective review. Dis Esophagus. 2011;24(5):3259. DOI: $10.1111 / \mathrm{j} .1442-2050.2010 .01144 . x$.

5. Iscan Y, Tunca F, Senyurek YG, Tezelman S, Terzioglu T. Thoracoscopic enucleation of a giant leiomyoma of the esophagus. Surg Laparosc Endosc Percutan Tech. 2013;23(1):e32-4. DOI: $10.1097 /$ SLE.0b013e318275b2a3.

6. Markakis CG, Spartalis ED, Liarmakopoulos E, Kavoura EG, Tomos P. Esophageal gastrointestinal stromal tumor: diagnostic complexity and management pitfalls. Case Rep Surg. 2013;2013:968394. DOI: 10.1155/2013/968394.
7. How CH, Lee JM. The robotic approach for enucleation of a giant esophageal lipoma. J Robot Surg. 2016;11(2):267-9. DOI: 10.1007/s11701-016-0668-7.

8. Luh SP, Hou SM, Fang CC, Chen CY. Video-thoracoscopic enucleation of esophageal leiomyoma. World J Surg Oncol. 2012;10:52. DOI: 10.1186/1477-7819-10-52.

9. Macke RA, Luketich JD, Nason KS, Schuchert MJ. Minimally invasive enucleation of a large, extensively calcified esophageal leiomyoma. J Thorac Cardiovasc Surg. 2014;147(4):e524. DOI: 10.1016/j.jtcvs.2013.12.054.

10. Khalaileh A, Savetsky I, Adileh M, Elazary R, Abu-Gazala M, Abu Gazala S, et al. Robotic-assisted enucleation of a large lower esophageal leiomyoma and review of literature. Int J Med Robot. 2013;9(3):253-7. DOI: 10.1002/rcs.1484.

11. Wang YX, Zhang J, Liu Y, Liu Y, Chu XY, Lu ZS, et al. Diagnosis and comprehensive treatment of esophageal leiomyoma: clinical analysis of 77 patients. Int J Clin Exp Med. 2015;8(10):17214-20.

12. Robb WB, Bruyere E, Amielh D, Vinatier E, Mabrut JY, Perniceni $\mathrm{T}$, et al. Esophageal gastrointestinal stromal tumor: is tumoral enucleation a viable therapeutic option? Ann Surg. 2015;261(1):117-24. DOI: 10.1097/SLA.0000000000000505.

13. Li QY, Meng Y, Xu YY, Zhang Q, Cai JQ, Zheng HX, et al. Comparison of endoscopic submucosal tunneling dissection and thoracoscopic enucleation for the treatment of esophageal submucosal tumors. Gastrointest Endosc. 2017;86(3):485-91. DOI: $10.1016 /$ j.gie.2016.11.023

14. Elli E, Espat NJ, Berger R, Jacobsen G, Knoblock L, Horgan S. Robotic-assisted thoracoscopic resection of esophageal leiomyoma. Surg Endosc. 2004;18(4):713-6. DOI: 10.1007/ s00464-003-8829-y.

15. Chen X, Xi Y, Wang H, Tan L. Minimally invasive surgery for giant esophageal leiomyoma: a case report \& review of the literatures. J Thorac Dis. 2017;9(1):E26-31. DOI: 10.21037/ jtd.2017.01.34.

16. Jeon HW, Choi MG, Lim CH, Park JK, Sung SW. Intraoperative esophagoscopy provides accuracy and safety in videoassisted thoracoscopic enucleation of benign esophageal submucosal tumors. Dis Esophagus. 2015;28(5):437-41. DOI: 10.1111/dote. 12220 .

17. Kumar A, Asaf BB. Robotic thoracic surgery: the state of the art. J Minim Access Surg. 2015;11(1):60-7. DOI: 10.4103/0972-9941.147693.

18. Compean SD, Gaur P, Kim MP. Robot assisted thoracoscopic resection of giant esophageal leiomyoma. Int J Surg Case Rep. 2014;5(12):1132-4. DOI: 10.1016/j.ijscr.2014.11.003.

19. Kocher GJ. Robotic-assisted thoracoscopic surgery: state of the art and future perspectives. J Thorac Dis. 2017;9(7):18557. DOI: $10.21037 /$ jtd.2017.06.139.

20. Shin S, Choi YS, Shim YM, Kim HK, Kim K, Kim J. Enucleation of esophageal submucosal tumors: a single institution's experience. Ann Thorac Surg. 2014;97(2):454-9. DOI: 10.1016/j.athoracsur.2013.10.030. 